\title{
BIVARIATE CARDINAL INTERPOLATION BY SPLINES ON A THREE-DIRECTION MESH
}

\author{
BY \\ Carl de Boor ${ }^{1}$, Klaus Hóllig $^{1,2}$ and Sherman Riemenschneider ${ }^{3}$ \\ Dedicated to I.J. Schoenberg \\ to whose insight and sense of beauty we are all indebted
}

\section{Introduction}

In this paper, we carry Schoenberg's beautiful cardinal spline theory $\left[\mathrm{S}_{2}\right]$, $\left[\mathrm{S}_{3}\right]$ over to a two-dimensional context which is not just the tensor product of the univariate situation. We find that we must work harder, yet must be satisfied with less precise results.

We are after a bounded cardinal interpolant to bounded data. This means that we are looking for a function of the form

$$
\text { If }=\sum_{j \in \mathbf{Z}^{2}} a_{j} M(\cdot-j)
$$

with $a \in l_{\infty}\left(\mathbf{Z}^{2}\right)$ which agrees with a given bounded function $f$ on $\mathbf{Z}^{2}$. Here, $M$ is a fixed function of compact support. In Section 2, we follow Schoenberg $\left[\mathrm{S}_{1}\right]$ in describing necessary and sufficient conditions on the Fourier transform of $M$ to insure the correctness of the interpolation problem, i.e., the existence and uniqueness of solutions.

We are particularly interested in using for $M$ a box spline, i.e., the twodimensional "shadow" of an $m$-dimensional cube, as given explicitly in (1) below. Let $Z$ be a set of vectors in $\mathbf{R}^{2}$. We find it convenient to change the definition $\left[\mathrm{BH}_{1}\right]$

$$
M \phi:=\int_{[0,1]^{Z}} \phi\left(\sum_{\zeta \in Z} \lambda(\zeta) \zeta\right) d \lambda
$$

of the box spline $M=M_{Z}$ to include an appropriate shift which makes the

\footnotetext{
Received April 12, 1983.

${ }^{1}$ Sponsored by a contract from the United States Army.

${ }^{2}$ Partially supported by a grant from the National Science Foundation.

${ }^{3}$ Supported by a grant from the NSERC Canada.
} 
origin the center of the support of $M$. This means that we use the definition

$$
M \phi:=\int_{[-1 / 2,1 / 2]^{z}} \phi\left(\sum_{\zeta \in Z} \lambda(\zeta) \zeta\right) d \lambda
$$

This gives the Fourier transform $\hat{M}$ of $M$ the symmetric form

$$
\hat{M}(x)=\prod_{\zeta \in Z} S\left(\zeta^{*} x\right)
$$

with

$$
S(t):=\frac{\sin t / 2}{t / 2}
$$

It is obvious from this formula that $M=M_{Z}$ is unchanged if one or more of the $\zeta \in Z$ are replaced by their negative; i.e.,

$$
M_{A Z}=M_{Z}
$$

if $A=\operatorname{diag}( \pm 1, \ldots, \pm 1)$. Further, if $A$ is any matrix, then

$$
\hat{M_{A Z}}(x)=\hat{M_{Z}}\left(A^{*} x\right) \text { and } \quad M_{A Z}(A x)=M_{Z}(x) / \operatorname{det} A .
$$

This allows one to deduce symmetries in $\hat{M}$ in case $A Z$ equals $Z$ after, possibly, some elements of $A Z$ have been multiplied by -1 .

The set $Z$ of directions can, of course, be chosen arbitrarily. But since we are interested in having

$$
\mathbf{S}:=\operatorname{span}(M(\cdot-j))_{j \in \mathbf{Z}^{2}}
$$

be a simple piecewise polynomial space, we choose $Z$ from $\mathbf{Z}^{2}$. It is shown in $\left[\mathrm{BH}_{1}\right]$ that the integer translates $M(\cdot-j), j \in \mathbf{Z}^{2}$, of the box spline are linearly dependent (when allowing for infinite linear combinations) in case the direction set $Z$ contains two vectors which span a proper sublattice of $\mathbf{Z}^{2}$. Linear independence is an obvious necessary condition for the cardinal interpolation problem to be correct. Thus, up to obvious symmetries, this leaves the three vectors $(1,0),(0,1)$ and $(1,1)$ as the only candidates for the directions $\zeta$ in $Z$.

With this restriction, $\mathbf{S}$ is a space of piecewise polynomial functions, of polynomial degree $|Z|-2$ or less, and with possible discontinuities only across the three types

$$
x(1)=k, \quad x(2)=k, \quad x(1)-x(2)=k, \quad k \in \mathbf{Z}
$$


of mesh lines. The overall smoothness of the elements of $\mathbf{S}$ depends on the multiplicities of the directions in $Z$. Such details, as well as the relationship of $\mathbf{S}$ to the space of all piecewise polynomial functions on such a three-direction mesh, of degree $|Z|-2$ and of specified smoothness, are all discussed in $\left[\mathrm{BH}_{2}\right]$.

In Section 3, we supply certain details concerning symmetries of such a three-direction box spline and its Fourier transform. We prove the correctness of cardinal interpolation with such a box spline in Section 4. We spend the major effort of this paper in Section 5 where we prove that, under reasonable conditions, the cardinal interpolant If of any suitably smooth function $f$ converges to $f$ as $|Z| \rightarrow \infty$. Specifically, we prove such convergence under the condition that $f$ is the Fourier transform of some compactly supported measure, following entirely the path established by Schoenberg [S] who showed in the univariate case that such convergence could be had whenever $\operatorname{supp} f$ $\subseteq(-\pi, \pi)$. We find, though, that, in our bivariate setup, there are many different sets playing the role of this interval, and which of these sets is relevant depends on the manner in which $|Z|$ goes to infinity.

The final section is devoted to the many detailed estimates on which the arguments in Section 5 are based.

\section{Cardinal interpolation}

Let $M: \mathbf{R}^{2} \rightarrow \mathbf{R}$ be a continuous function with compact support, and denote by

$$
\mathbf{S}:=\mathbf{S}_{M}:=\operatorname{span}\left\{M(\cdot-j): j \in \mathbf{Z}^{2}\right\}
$$

the space generated by its integer translates. Cardinal interpolation with $M$ concerns inversion of the linear map

$$
\mathbf{S} \cap \mathbf{L}_{\infty} \rightarrow l_{\infty}:\left.f \mapsto f\right|_{\mathbf{z}^{2}} .
$$

We say that cardinal interpolation with $M$ is correct if this map is 1-1 and onto, hence boundedly invertible, and denote its inverse by $I_{M}$ or $I$. In other words, cardinal interpolation with $M$ is correct iff there exists, for every bounded sequence $f \in l_{\infty}\left(\mathbf{Z}^{2}\right)$, a bounded function If $\in \mathbf{S}$ which agrees with $f$ on $\mathbf{Z}^{2}$. The interpolation problem, i.e., the determination of $I f$, is equivalent to the algebraic problem of determining the coefficient sequence $a$ for $I f=$ $\sum a_{j} M(\cdot-j)$ so that $a \in l_{\infty}$ and $\sum a_{j} M(\cdot-j)=f$ on $\mathbf{Z}^{2}$. Hence the correctness of cardinal interpolation is equivalent to the invertibility of the matrix

$$
A:=(M(j-k))_{j, k \in \mathbf{Z}^{2}}
$$

as a map on $l_{\infty}$. Since $A$ is a banded (bivariate) Toeplitz matrix, we have the 
following necessary and sufficient condition for the correctness of cardinal interpolation.

THEOREM 2. Cardinal interpolation with $M$ is correct iff

$$
P(x):=P_{M}(x):=\sum M(j) e^{i j x}
$$

does not vanish.

Proof. If $P(x)=0$, then $\left(e^{-i j x}\right)_{j \in \mathbf{Z}^{2}} \in(\operatorname{ker} A) \cap l_{\infty}$, and this contradicts the assumption that $A$ is 1-1. On the other hand, if $P$ does not vanish, then the inverse of $A$ can be expressed as a Toeplitz matrix,

$$
\left(A^{-1}\right)_{j k}:=\int_{(-\pi, \pi)^{2}} \frac{e^{-i(j-k) x}}{P(x)} d x / 2 \pi .
$$

In view of the geometric decay of the Fourier series for $1 / P$, we have

$$
\mid\left(A^{-1}\right)_{j k \mid} \leq \text { const } \lambda^{|j-k|}
$$

for some $\lambda=\lambda(P) \in(0,1)$. Therefore, $A^{-1}$ is bounded on $l_{p}\left(\mathbf{Z}^{2}\right)$ for any $p \in[1, \infty]$.

It is convenient to write the cardinal interpolant in Lagrange form:

$$
\text { If }=\sum f_{j} L(\cdot-j)
$$

with

$$
L:=L_{M}:=I \delta=\sum\left(A^{-1}\right)_{0 j} M(\cdot-j)
$$

the fundamental function of the interpolation process. The Fourier transform $L^{\wedge}$ of $L$ is particularly simple. Combining (4) with (6), we obtain

$$
L^{\hat{N}}=\hat{M} / P \text {. }
$$

We will also make use of the identity

$$
P(x)=\sum M^{\wedge}(2 \pi j-x)
$$

which follows from applying the Poisson summation formula $\sum f(j)=$ $\sum \hat{f}(2 \pi j)$ to (3).

\section{Cardinal interpolation with a box spline}

In this section, we develop in some detail facts about cardinal interpolation with the box spline $M_{Z}$. Recall from Section 1 that $(M(\cdot-j))_{j \in \mathbf{Z}^{2}}$ is linearly 
dependent if $Z$ contains two vectors which span a proper sublattice of $\mathbf{Z}^{2}$. Linear independence of $(M(\cdot-j))$ is an obvious necessary condition for cardinal interpolation with $M_{Z}$ to be correct. Thus, up to obvious symmetries, the only relevant case to consider is the case when the only directions in $Z$ are

$$
d_{1}:=(1,0), \quad d_{2}:=(0,1), \quad \text { and } \quad d_{3}:=(1,1) .
$$

We show in Section 4 that, with this restriction, cardinal interpolation with $M_{Z}$ is always correct.

Assume from now on that $Z=\left(d_{1}: r, d_{2}: s, d_{3}: t\right)$. In this case, $Z$ is characterized by the vector

$$
n:=\left(n_{1}, n_{2}, n_{3}\right):=(r, s, t)
$$

of direction multiplicities, and we will freely write $n$ instead of $Z$ whenever it is necessary to indicate by subscript the dependence on $Z$ of some quantity. Further, the general formulae given in Section 1 simplify. For example,

$$
M_{n}^{\hat{n}}(u, v)=S(u)^{r} S(v)^{s} S(u+v)^{t},
$$

with

$$
S(t):=\frac{\sin (t / 2)}{t / 2} .
$$

Further, the characteristic polynomial $P=P_{n}$ and the Fourier transform $L^{\wedge}$ of the fundamental spline $L=L_{n}$ have the representations

$$
\begin{aligned}
P(2 \pi u, 2 \pi v)= & \pi^{-|n|}(\sin (\pi u))^{r}(\sin (\pi v))^{s}(\sin (\pi(u+v)))^{t} \\
& \times \sum_{k, l} \frac{(-)^{r k+s l+t(k+l)}}{(u+k)^{r}(v+l)^{s}(u+v+k+l)^{t}}
\end{aligned}
$$

and

$1 / L^{\wedge}(2 \pi u, 2 \pi v)$

$$
=\sum_{k, l \in \mathbf{Z}}(-)^{r k+s l+t(k+l)}\left(\frac{u}{u+k}\right)^{r}\left(\frac{v}{v+l}\right)^{s}\left(\frac{u+v}{u+v+k+l}\right)^{t} .
$$

Let $A^{*}$ denote the transpose of $A$. The relation

$$
\hat{M_{A Z}}(x)=\hat{M_{Z}}\left(A^{*} x\right)
$$

valid for any matrix $A$ together with the fact that

$$
M_{A Z}=M_{Z}
$$


in case $A=\operatorname{diag}\left(\varepsilon_{1}, \ldots, \varepsilon_{|n|}\right)$ with $\varepsilon_{i} \in\{-1,1\}$, all $i$, implies certain symmetries of $M$ and $M^{\wedge}$ if the matrix $A$ leaves the set $Z_{ \pm}:=$ $\left\{d_{1}, d_{2}, d_{3},-d_{1},-d_{2},-d_{3}\right\}$ invariant. Denote by $\mathbf{A}$ the group of all such invertible matrices $A$. Each $A \in \mathrm{A}$ is associated with a permutation $\sigma_{A} \in \$_{3}$ (:= symmetric group on 3 elements) by the condition

$$
A d_{i} \in\left\{d_{\sigma_{A}(i)},-d_{\sigma_{A}(i)}\right\}, \quad i=1,2,3
$$

From the two matrices corresponding to a given $\sigma \in \$_{3}$, we choose one, $A_{\sigma}$, in such a way that the six matrices form a group and we call this group $\mathbf{A}_{+}$. Thus,

$$
A_{\sigma} d_{i} \in\left\{d_{\sigma(i)},-d_{\sigma(i)}\right\} \quad \text { for all } \sigma \in \$_{3},
$$

and one choice for the group generators are the three matrices

$$
A_{(12)}:=\left(\begin{array}{rr}
0 & -1 \\
-1 & 0
\end{array}\right), \quad A_{(13)}:=\left(\begin{array}{rr}
1 & 0 \\
1 & -1
\end{array}\right), \quad A_{(23)}:=\left(\begin{array}{rr}
-1 & 1 \\
0 & 1
\end{array}\right)
$$

corresponding to the transpositions (12), (13), and (23). With the definition

$$
\sigma(n):=\left(n_{\sigma(1)}, n_{\sigma(2)}, n_{\sigma(3)}\right) \text {, }
$$

it follows from (1.5) and from (4) that

$$
M_{n}(x)=M_{\sigma(n)}\left( \pm A_{\sigma} x\right), \text { and } \hat{M_{\sigma(n)}}(y)=\hat{M_{N}}\left( \pm A_{\sigma}^{*} y\right) .
$$

This implies

$$
P_{n}\left( \pm A_{\sigma}^{*} x\right)=P_{\sigma(n)}(x)
$$

and

$$
L_{n}(x)=\hat{L_{\sigma(n)}}\left( \pm A_{\sigma} x\right), \quad \hat{L_{\sigma(n)}}(y)=\hat{L_{n}}\left( \pm A_{\sigma}^{*} y\right)
$$

Of particular interest is the case $r=s=t$, i.e., when the direction multiplicities are all equal. In this case, $\sigma(n)=n$, all $\sigma$; i.e., (5)-(7) hold with $\sigma(n)$ replaced by $n$. For example, writing out in detail the relations (6) for $P=P_{(s, s, s)}$, we get

$$
P(u, v)=P(-u,-v)=P(v, u)=P(u+v,-v)=P(-u, u+v) .
$$

The relations for $\hat{M, \hat{P}}$ and $\hat{L}$ will be used frequently in the sequel. Since they are given in terms of the transposes of the matrices in $\mathbf{A}$, we now consider 


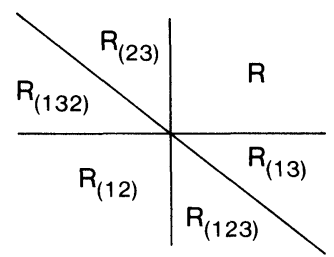

FIG. 3.1

$\mathbf{A}^{*}:=\left\{A^{*}: A \in \mathbf{A}\right\}$ in more detail. Set

$$
\begin{gathered}
d^{\prime}:=\left(\begin{array}{rr}
0 & 1 \\
-1 & 0
\end{array}\right) d \\
Z_{ \pm}^{\prime}:=\left\{d^{\prime}: d \in Z_{ \pm}\right\}=\{(0,1),(-1,0),(-1,1),(0,-1),(1,0),(1,-1)\} .
\end{gathered}
$$

Since $d^{\prime *} d=0$, we see from (4) that $\mathbf{A}^{*}$ leaves $Z_{ \pm}^{\prime}$ invariant. To further illustrate the action of the group $\mathbf{A}^{*}$, we divide $\mathbf{R}^{2}$ into the six cones $R_{\sigma}$, $\sigma \in \$_{3}$, as indicated in Figure 3.1. It is easily checked that

$$
A_{\sigma}^{*} R=R_{\sigma} \quad \text { for all } \sigma \in \$_{3} .
$$

\section{The correctness of cardinal interpolation with $M_{n}$}

In this section, we show that cardinal interpolation with $M_{n}$ is correct for all choices of $n \in \mathbf{Z}_{+}^{3}$.

TheOREM 4. For all $n \in \mathbf{Z}_{+}^{3}, P_{n}$ is strictly positive.

Since $P$ is $2 \pi$-periodic, this amounts to the claim that $P_{n}(x)>0$ for all $x \in[-\pi, \pi]^{2}$. This is the bivariate analogue of Schoenberg's well known result for univariate cardinal spline interpolation. To recall this result, denote by $N_{r}$ the univariate cardinal $B$-spline of degree $r$, and by $Q_{r}$ the corresponding characteristic polynomial given by

$$
Q_{r}(x):=\sum N_{r}(j) e^{i j x}
$$

Schoenberg showed in $\left[S_{1}\right]$ that

$$
\min _{x} Q_{r}(x)=Q_{r}(\pi)=2\left(\frac{2}{\pi}\right)^{r+1} \sum_{\nu=0}^{\infty} \frac{(-)^{\nu(r+1)}}{(2 \nu+1)^{r+1}}
$$

The fact that, for any $r$, the minimum is attained at $x=\pi$ is a consequence of 
the total positivity of the matrix $\left(N_{r}(j-k)\right)_{j, k \in \mathbf{z}}$. In view of this result, one might think that, in the above theorem, $\min _{u, v} P(u, v)=P(\pi, \pi)$. This is trivially true in the tensor product case, i.e., when $n=(r, s, 0)$. However, in general, the point at which $P_{n}$ attains its minimum depends on $n$. It would be interesting to determine its location for special choices of $n$. The nicest conjecture in this context (cf. Section 5) is that

$$
\min P(u, v)=P(2 \pi / 3,2 \pi / 3) \quad \text { if } n=(r, r, r) .
$$

In the proof of the theorem, we make use of (3.6). This allows us to assume without loss of generality that $r \geq s \geq t$.

We first consider two cases which reduce to Schoenberg's result.

The tensor product case $n=(r, s, 0)$. Here, we have $M_{n}(u, v)=N_{r}(u) N_{s}(v)$, and this implies that $P_{n}(u, v)=Q_{r}(u) Q_{s}(v)$.

The case $n=(r, 1,1)$. Since the open support of $M_{n}$ intersects exactly one mesh line of the form $(\cdot, l)$, viz. the meshline $(\cdot, 0)$, it follows that, in this case,

$$
M(k, l)=\left\{\begin{array}{ll}
N_{r}(k), & l=0, \\
0, & l \in \mathbf{Z} \backslash 0
\end{array} .\right.
$$

This means that cardinal interpolation with $M_{n}$ reduces to univariate interpolation with $N_{r}$ on each of the lines $(\cdot, l), l \in \mathbf{Z}$. In particular, $P(u, v)=Q_{r}(u)$.

For the proof of Theorem 4 , it remains to consider the cases where the multiplicities are all at least 1 , with equality for at most one. We make this assumption for the remainder of this section.

To prove the positivity of $P$, we use the representation $(2.10)$ in the form

$$
P(2 \pi x)=\sum \tilde{M}(x+j)
$$

with

$$
\tilde{M}(x):=\hat{M}(2 \pi x) \text {. }
$$

Recall from (3.1) that, for $x=(u, v)$ and $j=(k, l)$,

$$
\begin{aligned}
M^{\tilde{}}(x+j)= & \pi^{-|n|}(\sin \pi u)^{r}(\sin \pi v)^{s}(\sin \pi(u+v))^{t} \\
& \times \frac{(-)^{r k+s l+t(k+l)}}{(u+k)^{r}(v+l)^{s}(u+v+k+l)^{t}} .
\end{aligned}
$$

It is sufficient to show the positivity of $P(2 \pi \cdot)$ on $[0,1 / 2]^{2}$ for arbitrary $n$. This follows from (3.6) since, by (3.8), $[-1 / 2,1 / 2]^{2} \subseteq \cup_{A \in \mathbf{A}} A^{*}[0,1 / 2]^{2}$. For $x \in[0,1 / 2]^{2}$, we now show that the three positive terms

$$
\tilde{M}(x), \quad \tilde{M}\left(x-d_{1}\right), \text { and } \tilde{M}\left(x-d_{2}\right)
$$




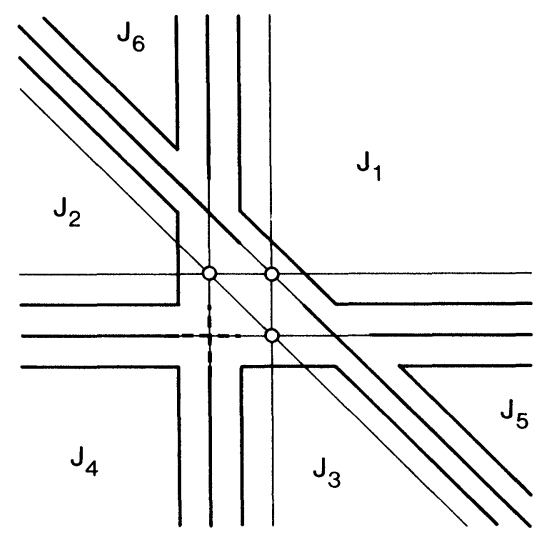

FIG. 4.1

dominate the sum in (3). To this end, we associate each of the other terms with one of these (even to the point of splitting one of the other terms between two of these) and show that each of the resulting three sums, when divided by their respective dominant term, is less than 1 . For ease of argument, we actually split the sum into altogether ten parts, as indicated in part by Fig. 4.1.

To simplify notation, we set

$$
b_{\nu}(j):=b_{\nu, n}(j, x):=\frac{|\tilde{M}(x+j)|}{\tilde{M}\left(x+j_{\nu}\right)}, \quad \nu=1,2,3,
$$

with

$$
j_{1}:=0, \quad j_{2}:=-d_{1}, \quad j_{3}:=-d_{2} .
$$

We now prove that

$$
\begin{gathered}
\sum_{j \in J_{1} \cup J_{4}} b_{1}(j)+\sum_{l \neq 0} b_{1}(l,-l)<1, \\
\sum_{j \in J_{2} \cup J_{5}} b_{2}(j)+\sum_{|l|>1} b_{2}(-1, l)+b_{2}(-1,-1) / 2<1, \\
\sum_{j \in J_{3} \cup J_{6}} b_{3}(j)+\sum_{|l|>1} b_{3}(l,-1)+b_{3}(-1,-1) / 2<1 .
\end{gathered}
$$

Since each of the summands (divided by its appropriate dominant term) other than the three dominant terms (5) occurs in (7)-(9) exactly once, we conclude from (7)-(9) the positivity of $P$.

The estimation of the various sums in (7)-(9) is straightforward. In each case, we find a majorant which is independent of $x \in[0,1 / 2]^{2}$ and $n$. For this, recall that we are assuming that $r, s, t \geq 1$ with at most one equality. 
We begin with the sum $\sum_{J_{1}} b_{1}(j)$. By (3.1) and (6), for $x=(u, v)$ and $j=(k, l)$ we have

$$
b_{1}(j)=\left|\frac{u}{u+k}\right|^{r}\left|\frac{v}{v+l}\right|^{s}\left|\frac{u+v}{u+v+k+l}\right|^{t} .
$$

Since $k, l \geq 0$ for $j=(k, l) \in J_{1}$ and we are assuming that $u, v \in[0,1 / 2]$, this quotient is largest when $u=v=1 / 2$; i.e.,

$$
b_{1}(j) \leq\left(\frac{1 / 2}{1 / 2+k}\right)^{r}\left(\frac{1 / 2}{1 / 2+l}\right)^{s}\left(\frac{1}{1+k+l}\right)^{t} .
$$

This bound is largest when the exponents $r, s, t$ are as small as possible, i.e., when $n=(1,2,2),(2,1,2)$ or $(2,2,1)$. Since

$$
\sum_{\substack{k, l \geq 0 \\ k, l) \neq 0}}\left(\frac{1 / 2}{1 / 2+k}\right)^{r}\left(\frac{1 / 2}{1 / 2+l}\right)^{s}\left(\frac{1}{1+k+l}\right)^{t}=.1723 \ldots
$$

for these values of $n$, we conclude that

$$
\sum_{J_{1}} b_{1}(j)<.18
$$

Similarly, one verifies that, for $j=(-k,-l) \in J_{4}$ and $x \in[0,1 / 2]^{2}$,

$$
b_{1}(j) \leq\left(\frac{1 / 2}{k-1 / 2}\right)^{r}\left(\frac{1 / 2}{l-1 / 2}\right)^{s}\left(\frac{1}{k+l-1}\right)^{t}
$$

and so obtains

$$
\sum_{J_{4}} b_{1}(j)<.02
$$

since

$$
\sum_{k, l>1}(2 k-1)^{-r}(2 l-1)^{-s}(k+l-1)^{-t}=.0101 \ldots
$$

for $(r, s, t)=(1,2,2),(2,1,2)$, or $(2,2,1)$.

Finally, for $j=(-l, l)$ and $l \neq 0$, we have

$$
b_{1}(-l, l) \leq \begin{cases}\left(\frac{1 / 2}{l+1 / 2}\right)^{r}\left(\frac{1 / 2}{l-1 / 2}\right)^{s}, & l>0 \\ \left(\frac{1 / 2}{-l-1 / 2}\right)^{r}\left(\frac{1 / 2}{-l+1 / 2}\right)^{s}, & l<0\end{cases}
$$


and so obtain

$$
\sum_{l \neq 0} b_{1}(l,-l) \leq \sum_{1}^{\infty}\left[\left(\frac{1 / 2}{l+1 / 2}\right)\left(\frac{1 / 2}{l-1 / 2}\right)^{2}+\left(\frac{1 / 2}{l+1 / 2}\right)^{2}\left(\frac{1 / 2}{l-1 / 2}\right)\right]=.5
$$

Combining (10)-(12) establishes (7).

The other inequalities are proved in a similar fashion and we only list the estimates involved.

Proof of (8).

$$
b_{2}(j)=\left|\frac{u-1}{u+k}\right|^{r}\left|\frac{v}{v+l}\right|^{s}\left|\frac{u+v-1}{u+v+k+l}\right|^{t} .
$$

The case $j=(-k, l) \in J_{2}$.

$$
b_{2}(j) \leq\left(\frac{1}{k}\right)^{r}\left(\frac{1 / 2}{l+1 / 2}\right)^{s}\left(\frac{1}{k-l}\right)^{t}
$$

and

$$
\sum_{k=2}^{\infty} \sum_{l=0}^{k-1}\left(\frac{1}{k}\right)^{r}\left(\frac{1 / 2}{l+1 / 2}\right)^{s}\left(\frac{1}{k-l}\right)^{t}=\left\{\begin{array}{l}
.25 \ldots \\
.23 \ldots \\
.30 \ldots
\end{array} \text { for }(r, s, t)=\left\{\begin{array}{l}
(2,2,1) \\
(2,1,2) \\
(1,2,2)
\end{array}\right.\right.
$$

Therefore $\sum_{J_{2}} b_{2}(j)<.35$.

The case $j=(k,-l) \in J_{5}$.

$$
b_{2}(j) \leq\left(\frac{1}{k}\right)^{r}\left(\frac{1 / 2}{l-1 / 2}\right)^{s}\left(\frac{1}{k-l}\right)^{t}
$$

and

$$
\sum_{k=3}^{\infty} \sum_{l=2}^{k-1}\left(\frac{1}{k}\right)^{r}\left(\frac{1 / 2}{l-1 / 2}\right)^{s}\left(\frac{1}{k-l}\right)^{t}=\left\{\begin{array}{l}
.027 \ldots \\
.081 \ldots \\
.010 \ldots
\end{array} \text { for }(r, s, t)=\left\{\begin{array}{l}
(2,2,1) \\
(2,1,2) \\
(1,2,2)
\end{array}\right.\right.
$$

Therefore $\sum_{J_{5}} b_{2}(j)<.1$. 
The case $j=(-1, l),|l|>1$.

$$
\begin{gathered}
b_{2}(j) \leq \begin{cases}\left(\frac{1 / 2}{l+1 / 2}\right)^{s}\left(\frac{1}{l-1}\right)^{t}, & l>1, \\
\left(\frac{1 / 2}{-l-1 / 2}\right)^{s}\left(\frac{1}{-l+1}\right)^{t}, & l<-1,\end{cases} \\
\sum_{|l|>1} b_{2}(j) \leq \sum_{l>1}\left[\left(\frac{1 / 2}{l+1 / 2}\right)\left(\frac{1}{l-1}\right)^{2}+\left(\frac{1 / 2}{l-1 / 2}\right)\left(\frac{1}{l+1}\right)^{2}\right]=.329 \ldots .
\end{gathered}
$$

The case $j=(-1,-1)$.

$$
b_{2}(-1,-1) \leq \frac{v}{1-v} \frac{1-u-v}{2-u-v} \leq 1 / 3 \quad(\text { at }(u, v)=(0,1 / 2)) .
$$

Proof of (9). Since $\hat{M_{(r, s, t)}}(u, v)=\hat{M_{(s, r, t)}}(v, u)$ and therefore

$$
b_{2,(r, s, t)}((u, v),(k, l))=b_{3,(s, r, t)}((v, u),(l, k)),
$$

the inequality (9) follows from (8) by interchanging the roles of $u$ and $v$ as well as those of $k$ and $l$.

This completes the proof of Theorem 4.

\section{Convergence of cardinal interpolation}

This section is devoted to the main goal of our paper, a study of the convergence of the cardinal interpolant to smooth functions as the degree tends to infinity. We prove the analogue of I.J. Schoenberg's basic result:

THEOREM $\left[\mathrm{S}_{2}\right]$. If $f$ is the Fourier transform of a measure with support in $(-\pi, \pi)$, then its cardinal spline interpolant $I_{r} f$ of degree $r$ converges to $f$ as the degree tends to infinity, i.e., $\left\|f-I_{r} f\right\|_{\infty} \rightarrow 0$ as $r \rightarrow \infty$.

This theorem is a consequence of the fact that the Fourier transform $\hat{L_{r}}$ of the fundamental spline converges to the characteristic function of the interval $(-\pi, \pi)$.

The bivariate situation is more complicated. Here, the limit of $\hat{L_{n}}$ depends on just how $n$ goes to infinity. Recall from (3.3) that

$$
1 / L^{\wedge}(2 \pi x)=1+\sum_{j \in \mathbf{Z}^{2} \backslash 0} \varepsilon_{j}(x) a_{n, j}(x),
$$

with $\varepsilon_{j}(x) \in\{-1,1\}$ and

$$
a_{n,(k, l)}(u, v):=\left|\frac{u}{u+k}\right|^{r}\left|\frac{v}{v+l}\right|^{s}\left|\frac{u+v}{u+v+k+l}\right|^{t} .
$$


Define the "middle component" $\mu(n)$ of $n$ by the requirement that it equal the middle or second number in any ordering of the three numbers $r, s, t$, and set

$$
n^{\prime}:=\left(r^{\prime}, s^{\prime}, t^{\prime}\right):=n / \mu(n) .
$$

Then, the typical summand in the right hand side of (1) is, up to sign,

$$
a_{n, j}(x)=\left(a_{n^{\prime}, j}(x)\right)^{\mu(n)} .
$$

This shows that $\hat{L}(2 \pi x)$ is close to 1 for large $\mu(n)$ provided $a_{n^{\prime}, j}(x)<1$ for all $j \neq 0$.

The set

$$
\left\{x: a_{n^{\prime}, j}(x)<1 \text { for all } j \in \mathbf{Z}^{2} \backslash 0\right\}
$$

depends on $n^{\prime}$. In particular, we cannot expect it to converge as $|n| \rightarrow \infty$ unless $n^{\prime}=n / \mu(n)$ converges, to some 3-vector $m$, say. Here, we are willing to allow $m$ to have infinite components. For example, if $n=\left(1, s, s^{2}\right)$, then $\mu(n)=s$ and $n^{\prime}=(1 / s, 1, s) \rightarrow(0,1, \infty)$ as $s \rightarrow \infty$. But not every $m \in[0, \infty]^{3}$ is a possible limit. By construction of $\mu(n), n^{\prime}=n / \mu(n)$ has exactly one component equal to 1 and, among the other two, one must be $\leq 1$ and the other must be $\geq 1$. Thus the set

$$
N:=\left\{n \in[0, \infty]^{3}: n_{\sigma(1)} \leq 1=n_{\sigma(2)} \leq n_{\sigma(3)} \text { for some } \sigma \in \$_{3}\right\}
$$

makes up the collection of all possible limits. On this set, we set up a topology of sorts by defining the open ball of radius $r$ around $m \in N$ by

$$
B_{r}(m):=\left\{n \in N: \max _{i}\left\{\begin{array}{ll}
\left|n_{i}-m_{i}\right|, & m_{i}<\infty \\
1 / n_{i}, & m_{i}=\infty
\end{array}\right\}<r\right\} .
$$

We extend the definition (2) of $a_{n, j}$ to all $n \in N$, by pointwise limit if necessary.

In what is to follow, the sets

$$
\Omega_{m}:=\left\{x: a_{m, j}(x)<1 \text { for } j \in J\right\},
$$

with

$$
J:=Z_{ \pm}^{\prime}=\{(1,0),(0,1),(-1,1),(-1,0),(0,-1),(1,-1)\}
$$

play a major role. Note that $\Omega_{n}=\Omega_{n^{\prime}}$. A qualitatively correct picture of $\Omega_{n}$ is given in Figure 5.1 which shows the roughly hexagonal shape of $\Omega_{n}$ and also 


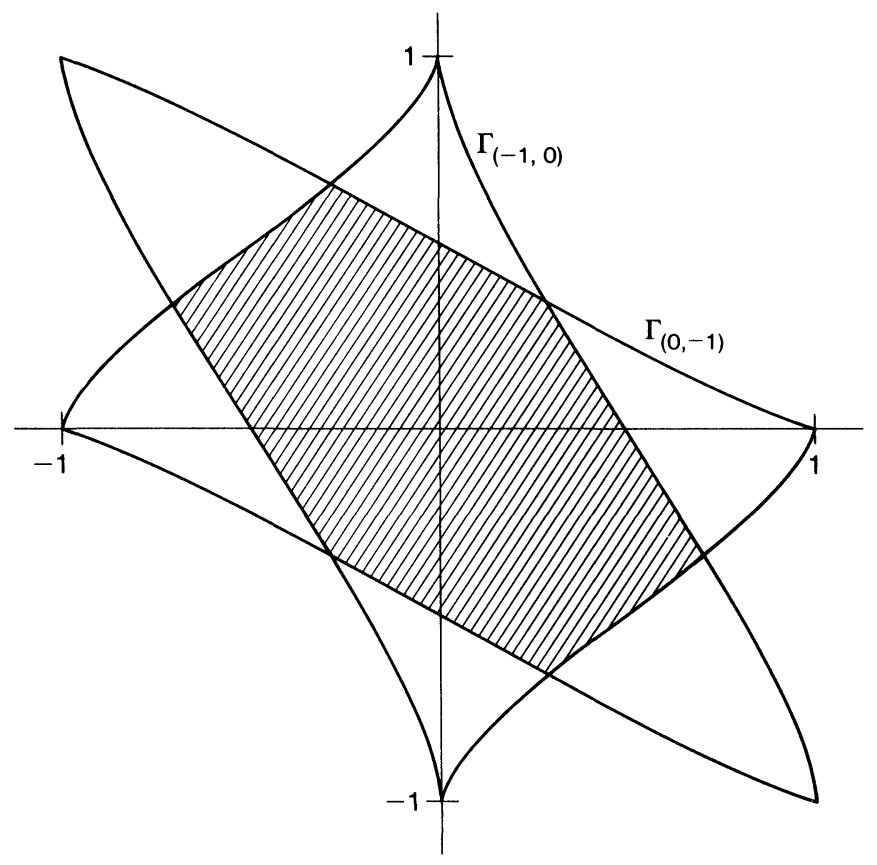

FIG. 5.1

shows the six curves

$$
\Gamma_{n, j}:=\left\{x \in C_{-j}: a_{n, j}(x)=1\right\}, \quad j \in J
$$

which contribute to the boundary, $\partial \Omega_{n}$. Here, $C_{-j}$ is the union of the two cones $R_{\sigma}$ which contain $-j$.

THEOREM 5.1. For $m \in N$, let $\chi_{m}$ be the characteristic function of $\Omega_{m}$. Then, for any $d>0$, there exists $\varepsilon>0$ so that

$$
\left|L_{n}^{\hat{n}}(2 \pi x)-\chi_{m}(x)\right| \leq C_{1}\left(1+C \operatorname{dist}\left(x, \partial \Omega_{m}\right)\right)^{-\mu(n)}
$$

for all $x$ with $\operatorname{dist}\left(x, \partial \Omega_{m}\right) \geq d$ and all $n \in(0, \infty)^{3}$ with $n^{\prime}:=n / \mu(n) \in B_{\varepsilon}(m)$, and with the positive constants $C_{1}$ and $C$ independent of $m, n, d$, or $x$.

Proof. The proof is based on a series of propositions which we merely state as needed and prove at leisure later. We begin with the following:

Proposition 5.1. $\Omega_{n}$ depends continuously on $n$ in the Hausdorff topology.

This is part of the corollary to Lemma 6.4 below. It implies, given $d>0$, the existence of $\varepsilon>0$ so that $\operatorname{dist}\left(\Omega_{n^{\prime}}, \Omega_{m}\right) \leq d / 2$ for all $n^{\prime} \in B_{\varepsilon}(m)$. Conse- 
quently,

$$
\operatorname{dist}\left(x, \partial \Omega_{m}\right) \leq 2 \operatorname{dist}\left(x, \partial \Omega_{n^{\prime}}\right)
$$

for all $x$ with $\operatorname{dist}\left(x, \partial \Omega_{m}\right) \geq d$ and for all $n^{\prime} \in B_{\varepsilon}(m)$. It is therefore sufficient to prove (3) with $m$ replaced by $n^{\prime}$.

For its proof, we use (1) and we consider two cases.

(i) $x \in \Omega_{n^{\prime}}$. We need:

Proposition 5.2. Let

$$
J^{\prime}:=\mathbf{A}^{*}(1,1)=\{ \pm(1,1), \pm(2,-1), \pm(-1,2)\}
$$

For $n \in N$ and $x \in \Omega_{n^{\prime}}=\Omega_{n}$,

$$
a_{n, j}(x) \leq \begin{cases}\left(1+C \operatorname{dist}\left(x, \partial \Omega_{n}\right)\right)^{-1}, & j \in J \cup J^{\prime} \\ (1+C|j|)^{-1}, & j \in \mathbf{Z}^{2} \backslash\left(0 \cup J \cup J^{\prime}\right)\end{cases}
$$

with the positive constant $C$ independent of $n, j$, or $x$.

This, together with (1), implies that

$$
\begin{aligned}
\left|1 / \hat{L_{n}^{\hat{n}}}(2 \pi x)-1\right| & \leq \sum_{j \neq 0}\left(a_{n^{\prime}, j}(x)\right)^{\mu(n)} \\
& \leq 12\left(1+C \operatorname{dist}\left(x, \partial \Omega_{n^{\prime}}\right)\right)^{-\mu(n)}+\sum_{j \notin 0 \cup J \cup J^{\prime}}(1+C|j|)^{-\mu(n)} \\
& \leq C_{1}\left(1+C \operatorname{dist}\left(x, \partial \Omega_{n^{\prime}}\right)\right)^{-\mu(n)}
\end{aligned}
$$

and so proves (3) for this case.

(ii) $x \notin \Omega_{n^{\prime}}$. For this case, we need:

Proposition 5.3. The integer translates of $\Omega_{n}$ form, up to a set of measure zero, a partition of $\mathbf{R}^{2}$; i.e.,

$$
\mathbf{R}^{2}=\bigcup_{j \in \mathbf{Z}^{2}} j+\Omega_{n}^{-}, \quad \Omega_{n} \cap\left(j+\Omega_{n}\right)=\emptyset \quad \text { for } j \neq 0
$$


We conclude that there is $j \neq 0$ so that $x=x^{\prime}+j$ with $x^{\prime} \in \Omega_{n^{\prime}}^{-}$. With this, we use the periodicity of the characteristic polynomial $P$ to write for such $x$,

$$
\begin{aligned}
\hat{L^{\wedge}(2 \pi x)} & =\hat{L^{\wedge}}\left(2 \pi\left(x^{\prime}+j\right)\right) \\
& =\frac{\hat{M}\left(x^{\prime}+j\right)}{P\left(2 \pi\left(x^{\prime}+j\right)\right)} \frac{\tilde{M}\left(x^{\prime}\right)}{M^{\tilde{}}\left(x^{\prime}\right)} \\
& =\hat{L^{\wedge}}\left(2 \pi x^{\prime}\right) \frac{M^{\tilde{}}\left(x^{\prime}+j\right)}{M^{\tilde{}}\left(x^{\prime}\right)}
\end{aligned}
$$

Therefore

$$
\left|\hat{L_{n}}(2 \pi x)\right|=\left|\hat{L_{n}}\left(2 \pi x^{\prime}\right)\right| a_{n, j}\left(x^{\prime}\right)=\left|\hat{L_{n}}\left(2 \pi x^{\prime}\right)\right|\left(a_{n^{\prime}, j}\left(x^{\prime}\right)\right)^{\mu(n)} .
$$

By (5), $\left|\hat{L_{n}}\left(2 \pi x^{\prime}\right)\right| \leq C$ since $x^{\prime} \in \Omega_{n^{\prime}}^{-}$. Thus (3) is proved for this case once we show:

Proposition 5.4. Let $x=x^{\prime}+j$ with $j \in \mathbf{Z}^{2} \backslash 0$ and $x^{\prime} \in \Omega_{n^{\prime}}^{-}$, and with $n \in \mathbf{R}_{+}^{3}$. Then

$$
a_{n^{\prime}, j}\left(x^{\prime}\right) \leq\left(1+C \operatorname{dist}\left(x, \partial \Omega_{n^{\prime}}\right)\right)^{-1}
$$

for some positive constant $C$ independent of $n$ and $x$.

This finishes the proof of Theorem 5.1.

THEOREM 5.2. Let $f$ be the Fourier transform of a measure with support strictly inside $2 \pi \Omega_{m}$ for some $m \in N$; i.e.,

$$
d:=\operatorname{dist}\left(\operatorname{supp} \hat{f}, \partial\left(2 \pi \Omega_{m}\right)\right)>0 .
$$

Then there exists $\varepsilon>0$ so that, for all $n \in B_{\varepsilon}(m)$,

$$
\left\|f-I_{n} f\right\|_{\infty} \leq C_{1}(1+C d)^{-\mu(n)}\left\|f^{\wedge}\right\|_{1}
$$

with $\left\|f^{\wedge}\right\|_{1}$ the total variation of $f^{\wedge}$. The positive constants $C, C_{1}$ do not depend on $m, d$, or $n$.

Proof. Fix $d>0$ and choose $\varepsilon>0$ so that

$$
\operatorname{supp} \hat{f \subseteq}\left(2 \pi \Omega_{n^{\prime}}\right) \text { and } \operatorname{dist}\left(\operatorname{supp} \hat{f,} \partial\left(2 \pi \Omega_{n^{\prime}}\right)\right) \geq d / 2
$$


for all $n^{\prime} \in B_{\varepsilon}(m)$. We have to estimate

$$
f(x)-\sum f(j) L_{n}(x-j)=f(x)-\sum(2 \pi)^{-2} \int_{\mathbf{R}^{2}} f(j) e^{-i j y} L_{n}^{\hat{n}}(y) e^{i x y} d y .
$$

Since $2 \pi \Omega_{n^{\prime}}$ is a fundamental domain, i.e., translates by its $2 \pi j, j \in \mathbf{Z}^{2}$, form a partition of unity (by Proposition 5.3), and supp $\hat{f} \subseteq 2 \pi \Omega_{n^{\prime}},(f(j))_{j \in \mathbf{Z}^{2}}$ are the Fourier coefficients of the periodic extension $f_{p}^{\prime}$ of the measure $f$. Using the weak convergence of the Fourier series of a measure, we obtain

$$
f(x)-\left(I_{n} f\right)(x)=(2 \pi)^{-2} \int_{\mathbf{R}^{2}}\left[f^{\hat{n}}(y)-\hat{f_{p}}(y) \hat{L_{n}}(y)\right] e^{i x y} d y .
$$

Applying Theorem 5.1 yields, for $n^{\prime} \in B_{\varepsilon}(m)$,

$$
\begin{aligned}
& \left\|f-I_{n} f\right\|_{\infty} \\
& \leq(2 \pi)^{-2} C_{1}(1+C d)^{-\mu(n)}\left\|\hat{f^{\wedge}}\right\|_{1}+\sum_{j \neq 0}\left\|\hat{L_{n}}(\cdot+2 \pi j)\right\|_{\infty, \operatorname{supp} f^{\wedge}}\left\|\hat{f^{\wedge}}\right\|_{1} \\
& \leq\left[C_{1}(1+C d)^{-\mu(n)}+\sum_{j \notin 0 \cup J \cup J^{\prime}}(1+C|j|)^{-\mu(n)}\right]\left\|f^{\wedge}\right\|_{1} \\
& \leq C_{1}(1+C d)^{-\mu(n)}\left\|f^{\wedge}\right\|_{1} \text {. }
\end{aligned}
$$

We now discuss briefly the particularly symmetric and special case $n=$ $(r, r, r)$. Figure 5.2 shows the level lines for $P_{r}:=P_{(r, r, r)}$ for $r=3$. Note that its minimum seems to occur at $(2 \pi / 3,2 \pi / 3)$, and this can be verified analytically for $r<4$. We conjecture that this is no accident, but is the case for all $r$.

Figure 5.3 shows $L_{r}:=L_{(r, r, r)}$ for $r=3$. The fast decay is quite striking, making plain that cardinal interpolation with this $L$ would be strongly essentially local.

If we assume that $\hat{f} \in \mathbf{L}_{2}$ with supp $\hat{f} \subseteq 2 \pi \Omega_{m}$, then the convergence of $I_{n} f$ can be stated in a particularly nice way. We define a bivariate "Whittaker" operator

$$
W: l_{2} \rightarrow \mathbf{L}_{2}: f \mapsto \sum_{j \in \mathbf{Z}^{2}} f(j) \chi^{\wedge}(\cdot-j),
$$

with

$$
\begin{aligned}
& \chi(u, v):= \\
& \frac{9}{2 \pi^{2}}\left[\frac{\cos \frac{2 \pi}{3}(u+v)}{(u-2 v)(v-2 u)}+\frac{\cos \frac{2 \pi}{3}(2 u-v)}{(u+v)(u-2 v)}+\frac{\cos \frac{2 \pi}{3}(2 v-u)}{(u+v)(v-2 u)}\right]
\end{aligned}
$$




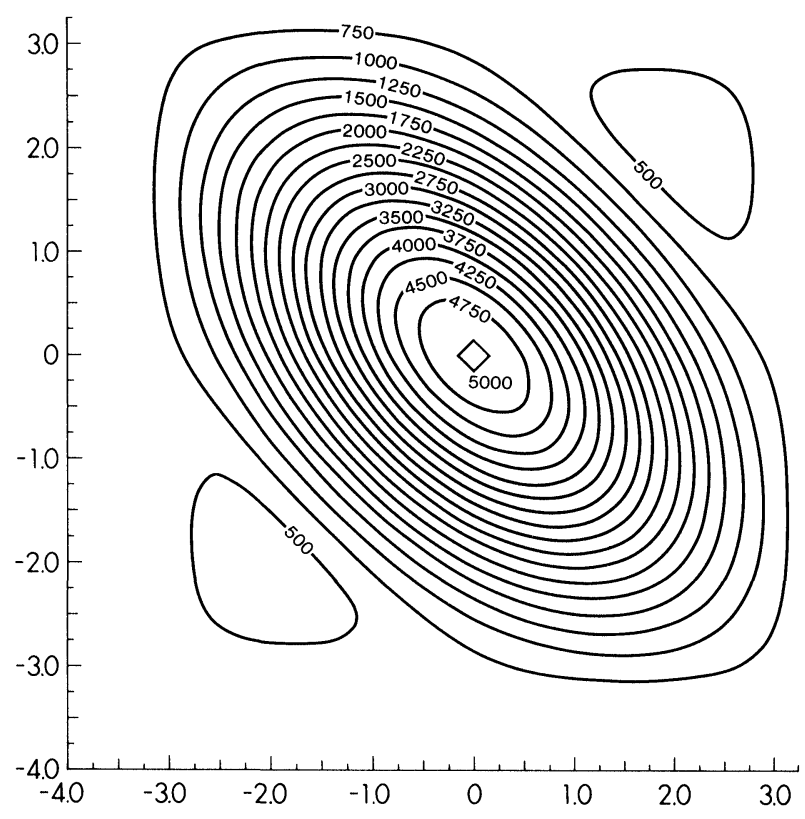

FIG. 5.2

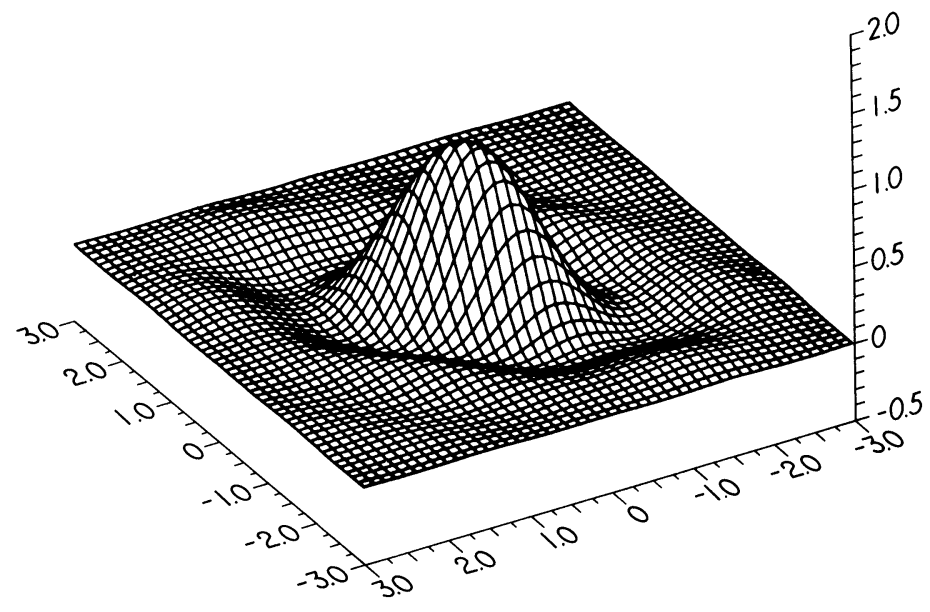

FIG. 5.3 
the Fourier transform of $\chi:=\chi_{\Omega}, \Omega:=2 \pi \Omega_{(1,1,1)}$. Note that the translates of $\chi$ are orthogonal in $\mathbf{L}_{2}$. As in the univariate case [ $\left.\mathrm{S}_{2}\right]$, the "Whittaker" series provides the limiting operator for cardinal interpolation $I_{(r, r, r)}$ as $r \rightarrow \infty$. More precisely, we have

$$
\left\|W-I_{r}: l_{2} \rightarrow \mathbf{L}_{2}\right\| \rightarrow 0 \quad \text { as } r \rightarrow \infty .
$$

If $f \in \mathbf{L}_{2}$ and $\operatorname{supp} \hat{f} \subseteq 2 \pi \Omega_{(1,1,1)}$, we have $W\left((f(j))_{\mathbf{z}^{2}}\right)=f$ and hence (7) is an $\mathbf{L}_{2}$-version of Theorem 5.2.

To prove (7), we first show that the cardinal interpolation maps $I_{r}:=I_{(r, r, r)}$ are bounded as maps from $l_{2}$ to $\mathbf{L}_{2}$ uniformly in $r$ : For $f \in l_{2}$, we have

$$
\left\|I_{r} f\right\|_{2}=\left\|\sum f(j) e^{-i j} \cdot L_{r}^{\wedge}\right\|_{2} \leq\|f\|_{2}\left(\sum\left\|L_{r}^{\hat{r}}(\cdot+2 \pi j)\right\|_{\infty, \Omega}^{2}\right) \leq C\|f\|_{2} .
$$

By the uniform boundedness principle, it is therefore sufficient to check the convergence (7) for the unit vector $e_{j} \in l_{2}$. But this is an obvious consequence of (3).

In the univariate setting, [MRR] proved the convergence in $\mathbf{L}_{p}$ for all $p \in(1, \infty)$, but we leave the corresponding bivariate problem to a later paper.

[R] extended Schoenberg's univariate result to include the possibility that $f^{\wedge}$ has support at $\pm \pi$. This requires the realization that $\hat{L_{r}}( \pm \pi) \rightarrow 1 / 2$. Theorem 5.1 says nothing about the limit of $\hat{L_{r}}(x)$ in case $x \in \partial\left(2 \pi \Omega_{m}\right)$. For the special choice $n=(r, r, r)$, such a statement is relatively easy to make.

COROLlary . For the special choice $n=(r, r, r)$,

$$
\lim _{r \rightarrow \infty} \hat{L_{n}}(x)= \begin{cases}1 / 3, & x \in \mathbf{A} z, \\ 1 / 2, & x \in \partial(2 \pi \Omega) \backslash \mathbf{A} z,\end{cases}
$$

with $z:=(2 \pi / 3,2 \pi / 3)$.

Clearly, our result concerning the convergence of $I_{n}$ still holds if $f^{\wedge}$ is a measure, absolutely continuous in a neighborhood of $\partial 2 \pi \Omega_{m}$ and supported in $2 \pi \Omega_{m}$. Our result is best possible in the following sense. If $\operatorname{supp} f \hat{\cap} \partial 2 \pi \Omega \neq \varnothing$, then, in general, $I_{n} f$ does not converge to $f$. For example, if $f(x)=\cos \left(z^{*} x\right)$, then

$$
\begin{aligned}
I_{r} f(x) & \underset{r \rightarrow \infty}{\rightarrow} \sum_{A \in \mathbf{A}} e^{i(A z)^{* x}} \\
& =\left[\cos \frac{2 \pi}{3}(u+v)+\cos \frac{2 \pi}{3}(2 u-v)+\cos \frac{2 \pi}{3}(2 v-u)\right] .
\end{aligned}
$$

This follows from the corollary. However, it requires slightly more precise 
information about the convergence asserted in (8). The heuristic argument is that

$$
\left(I_{n} f\right)^{\wedge}=f_{p} \hat{L}_{n}^{\hat{n}}=\frac{1}{2}(2 \pi)^{2} \sum \delta_{z+2 \pi j} \hat{L_{n}}(z+2 \pi j)
$$

and therefore

$$
I_{n} f \rightarrow \sum_{A \in \mathbf{A}^{*}}\left(\delta_{A z} \cdot 1 / 3\right) \text { as } n \rightarrow \infty .
$$

Here, $\delta_{\xi}$ denotes the Dirac measure at $\xi$.

\section{Detailed estimates}

In this section, we prove Propositions 5.1-5.4 and various lemmas needed in the proofs. This amounts to a detailed study of the functions $a_{n, j}$ and the set $\Omega_{n}$ and how they depend on $n \in N$. In particular, we need to study the boundary of $\Omega_{n}$. This boundary is made up of pieces of curves given implicitly by the equation $a_{n, j}(x)=1$ for some $j \in J$.

We use the symmetries of the given situation. Recall the notation

$$
\sigma(n):=\left(n_{\sigma(1)}, n_{\sigma(2)}, n_{\sigma(3)}\right) .
$$

We conclude from (3.5) or directly from (5.2) that

$$
a_{n, j}(x)=a_{\sigma(n), A_{\sigma}^{*} j}\left(A_{\sigma}^{*} x\right) .
$$

This implies that

$$
\pm A_{\sigma}^{*} \Omega_{n}=\Omega_{\sigma(n)}
$$

and therefore

$$
A_{\sigma}^{*}\left(\Omega_{n} \cap R\right)=\Omega_{\sigma(n)} \cap R_{\sigma},
$$

where, to recall from Figure 3.1, $R=R_{(1)}=\mathbf{R}_{+}^{2}$.

Next, we consider the boundary of $\Omega_{n}$ (cf. Figure 5.1). Each $-j \in J$ lies in two cones $R_{\sigma}$. With $C_{-j}$ their union, we define the curve

$$
\Gamma_{n, j}:=\left\{x \in C_{-j}: a_{n, j}(x)=1\right\} .
$$

The boundary of $\Omega_{n}$ is made up of segments of these curves. It follows from (1) that

$$
A_{\sigma}^{*} \Gamma_{n, j}=\Gamma_{\sigma(n), A_{\sigma}^{*} j}
$$


LEMMA 6.1. For $j \in J$, denote by $j_{0}, j_{1}$ the vectors spanning the union $C_{j}$ of the two cones $R_{\sigma}$ containing $j$. For $n \in(0, \infty)^{3}$, the curve $\Gamma_{n, j}$ passes through the points $-j_{0},-j / 2,-j_{1}$ and is symmetric with respect to the point $-j / 2$. Moreover, it is monotone (in an appropriate coordinate system) and is Lipschitz continuous, uniformly in $n$.

Proof. The symmetry with respect to the point $-j / 2$ follows from the relations

$$
a_{n, j}(x-j)=1 / a_{n,-j}(x)=1 / a_{n, j}(-x)
$$

which are immediate consequences of the definition (5.2) of $a_{n, j}$. For the rest, it is, in view of (5), sufficient to consider $j=(-1,0)$. In this case, the curve $\Gamma_{n, j}$ is given by the equation

$$
\left|\frac{u}{u-1}\right|^{r}\left|\frac{u+v}{u+v-1}\right|^{t}=1
$$

Using the fact that $u \geq 0, u+v \geq 0$, and solving for $v$, we obtain

$$
v=-u+1 /\left[1+\left(\frac{u}{1-u}\right)^{\alpha}\right], \quad 0 \leq u \leq 1
$$

where $\alpha:=r / t$. This shows that, for any $\alpha \in(0, \infty)$, the points $-j_{0}=(1,-1)$, $-j / 2=(1 / 2,0)$, and $-j_{1}=(0,1)$ lie on the curve. Moreover we have

$$
d v / d u=-1-\left[1+\left(\frac{u}{1-u}\right)^{\alpha}\right]^{-2} \alpha\left(\frac{u}{1-u}\right)^{\alpha-1}(1-u)^{-2}
$$

which shows that

$$
d v / d u \leq-1
$$

with equality only if $u=0$. This proves the remaining assertions of the Lemma.

LEMMA 6.2. Let $j_{0}, j_{1}$ be the two vectors which span the cone $R_{\sigma}$. For $n \in(0, \infty)^{3}$, the curves $\Gamma_{n,-j_{0}}$ and $\Gamma_{n,-j_{1}}$ intersect at a unique point $z_{n, \sigma} \in R_{\sigma}$. The boundary of $\Omega_{n}$ consists of the segments of the curves $\Gamma_{n, j}$ connecting the points in $J / 2$ and $z_{n, \sigma}, \sigma \in \$_{3}$.

Proof. In view of (3) and (5), it is sufficient to consider the case $\sigma=(1)$, $R_{\sigma}=R$ and $j_{0}=(1,0), j_{1}=(0,1)$. By $(9), \Gamma_{n,(-1,0)}$ has slope $\leq-1$, with equality possible only at the point $(0,1)$. Similarly, a direct computation shows that $\Gamma_{n,(0,-1)}$ has slope between -1 and 0 . Since $\Gamma_{n,(-1,0)}$ and $\Gamma_{n,(0,-1)}$ pass 
through the points $(0,1),(1 / 2,0)$ and $(0,1 / 2),(1,0)$ respectively, they intersect at a unique point $z_{n,(1)} \in(0,1 / 2)^{2}$. To show that the boundary of $\Omega_{n}$ intersected with $R$ consists of the segments connecting $(0,1 / 2)$ with $z_{n,(1)}$, and $z_{n,(1)}$ with $(1 / 2,0)$, we prove that, for $x=(u, v) \in R$,

$$
a_{n,(-1,0)}(x), a_{n,(0,-1)}(x)<1
$$

implies that

$$
a_{n, j}(x)<1 \text { for all } j \in J
$$

Indeed,

$$
a_{n,(-1,0)}(x)=\left|\frac{u}{u-1}\right|^{r}\left|\frac{u+v}{u+v-1}\right|^{t}<1
$$

implies $u<1 / 2$, and, from $a_{n,(0,-1)}(x)<1$, it follows that $v<1 / 2$. This implies

$$
a_{n,(-1,1)}(x)=\left|\frac{u}{u-1}\right|^{r}\left|\frac{v}{v+1}\right|^{t}<1,
$$

and the other cases can be checked just as easily.

LemMA 6.3. For all $m \in N, \quad \Gamma_{m, J}=\lim _{n \rightarrow m}\left\{\Gamma_{n, j}: \quad n \in(0, \infty)^{3} \cap N\right\}$. Hence Lemma 1 is valid for all $n \in N$.

Proof. Without loss of generality, we consider only the case $j=(-1,0)$. We claim that

$$
\Gamma_{m, j}=\left\{\begin{array}{l}
B L((0,1),(0,1 / 2),(1 / 2,0),(1,-1 / 2),(1,-1)) \\
\quad \text { if } m_{1}=0 \text { and } / \text { or } m_{3}=\infty \\
B L((0,1),(1 / 2,1 / 2),(1 / 2,0),(1 / 2,-1 / 2),(1,-1)) \\
\quad \text { if } m_{1}=\infty \text { and } / \text { or } m_{3}=0
\end{array}\right.
$$

where $B L\left(x_{1}, \ldots, x_{m}\right)$ denotes the broken line with vertices $x_{1}, \ldots, x_{m}$. For example, consider the first case. By (9) and the symmetry of the curves $\Gamma_{n, j}$ and $\Gamma_{m, j}$, for $n \in(0, \infty)^{3}$ we have

$$
\operatorname{dist}\left(\Gamma_{m, j}, \Gamma_{n, j}\right) \leq u_{n},
$$

with $u_{n}$ such that $a_{n, j}\left(u_{n}, 1 / 2\right)=1$. From (6), we obtain

$$
\gamma:=t / r=\frac{\ln \left(1-u_{n}\right)-\ln u_{n}}{\ln \left(u_{n}+1 / 2\right)-\ln \left(1 / 2-u_{n}\right)} .
$$


If $n \rightarrow m$ with $m_{1}=0$ and/or $m_{3}=\infty$, we must have $\gamma \rightarrow \infty$ and, by the above equation, this implies that $u_{n} \rightarrow 0$. The second case can be handled similarly.

Lemma 3 is a particular case of the next lemma which states that $\Gamma_{n, j}$ depends continuously on $n$.

Lemma 6.4. For $n, m \in N$, $\operatorname{dist}\left(\Gamma_{n, j}, \Gamma_{m, j}\right) \rightarrow 0$ as $n \rightarrow m$.

Proof. In view of Lemma 3, we may assume that $m \in(0, \infty)^{3}$. Moreover, it is sufficient to consider $j=(-1,0)$. In this case, it follows from (7) that $\Gamma_{n, j} \rightarrow \Gamma_{m, j}$ pointwise, both curves being viewed as functions of $u$. By the uniform Lipschitz continuity of the curves, this implies the assertion of the lemma.

Lemmas 1-4 give a qualitative description of the boundary of $\Omega_{n}$. We summarize the main features in the following result.

Corollary. (i) $\partial \Omega_{n}$ consists of segments of the curves $\Gamma_{n, j}$ connecting the points in $J / 2$ with the intersection points $z_{n, \sigma}, \sigma \in \$_{3}$.

(ii) $\partial \Omega_{n}$ is piecewise monotone and is Lipschitz continuous, uniformly in $n$.

(iii) $\Omega_{n}$ depends continuously on $n$ in the Hausdorff topology.

Note that this provides the proof of Proposition 5.1.

To give a few examples, we list in Figs. 6.1-6.3 all cases for which $\Omega_{n}$ has a piecewise linear boundary. Moreover, we have

$$
\begin{aligned}
& \Omega_{(1,1,0)}=\Omega_{(\infty, 1,0)}=\Omega_{(1, \infty, 0)} \\
& \Omega_{(1,0,1)}=\Omega_{(\infty, 0,1)}=\Omega_{(1,0, \infty)} \\
& \Omega_{(0,1,1)}=\Omega_{(0, \infty, 1)}=\Omega_{(0,1, \infty)} .
\end{aligned}
$$

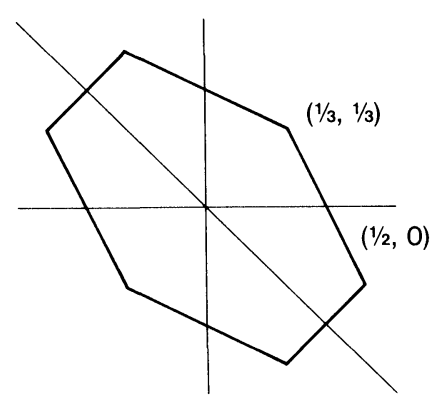

FIG. $6.1 n=(1,1,1)$ 

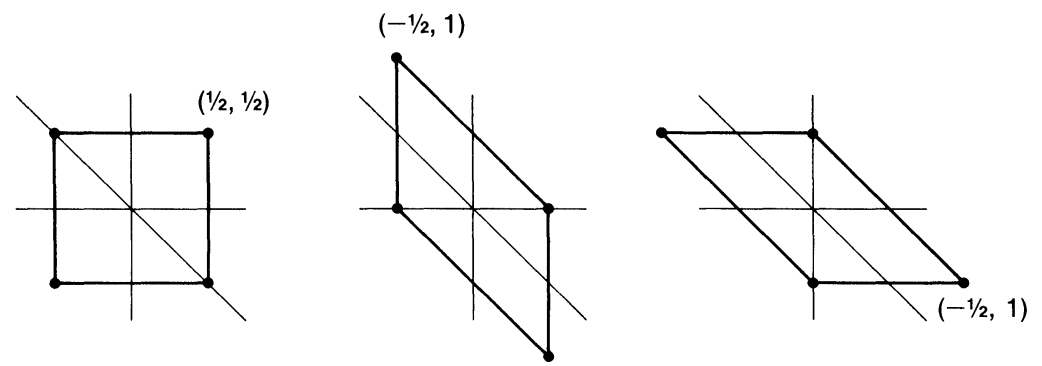

FIG. $6.2 n=(1,1,0),(1,0,1),(0,1,1)$
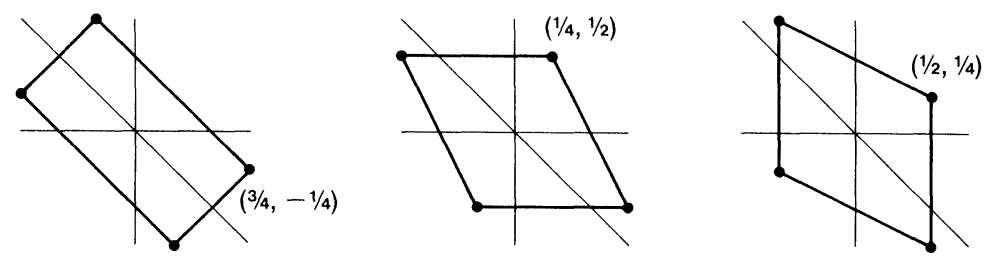

FIG. $6.3 n=(1,1, \infty),(1, \infty, 1),(\infty, 1,1)$

We take the occasion to prove the following observation which stresses the underlying hexagonal structure.

Proposition 6. $\Omega_{0}:=\cap_{n} \Omega_{n}=$ int $\operatorname{conv} J / 2, \quad \Omega_{1}:=\cup_{n} \Omega_{n}=\cup_{A \in \mathbf{A}_{+}}$ $A^{*}[0,1 / 2)^{2}$.

Proof. We claim that, for any $n$,

$$
\{(u, v): u, v \geq 0, u+v<1 / 2\} \subseteq R \cap \Omega_{n} \subseteq[0,1 / 2)^{2}
$$

This follows from Lemma 1, in particular from the fact that the curves $\Gamma_{n,(-1,0)}$ and $\Gamma_{n(0,-1)}$ pass through the points $(1 / 2,0)$ and $(0,1 / 2)$ and, as functions of $u$, have slopes $\leq-1$ and $\geq-1$, respectively. To complete the proof, note that $n=(0,1,1)$ gives equality in the first inclusion of $(11)$ while $n=(1,1,0)$ gives equality in the second.

We are now also ready for:

Proof of Proposition 5.3. Because of the continuity of $\Omega_{n}$ as a function of $n$, it is sufficient to consider $n \in(0, \infty)^{3}$. In this situation, Figure 5.1 gives a qualitatively correct description of $\Omega_{n}$. Because of the geometry of $\Omega_{n}$ and the 

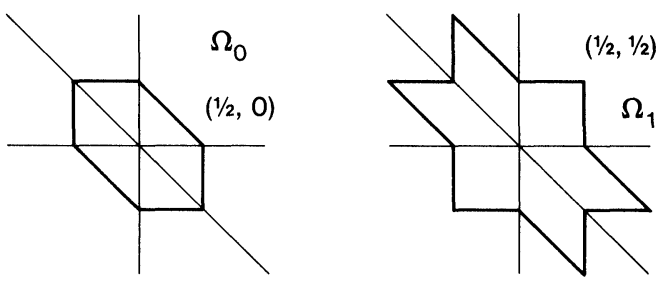

FIG. 6.4

symmetry relations (2), it is sufficient to establish the following claims:

(i) $j+\Gamma_{n, j}=\Gamma_{n,-j}$ for all $j \in J$.

(ii) The curve $(1,0)+\Gamma_{n,(1,-1)}$ passes through the point $z_{n,(1)}$.

The first assertion follows from the relation $a_{n, j}(x-j)=1 / a_{n,-j}(x)$ alluded to earlier and directly derivable from the definition (5.2) of $a_{n, j}$. As to (ii), note that

$$
a_{n,(-1,0)}\left(z_{n,(1)}\right)=1=a_{n,(0,-1)}\left(z_{n,(1)}\right)
$$

implies that

$$
1=a_{n,(0,-1)} / a_{n,(-1,0)}\left(z_{n,(1)}\right)=a_{n,(1,-1)}\left(z_{n,(1)}-(1,0)\right) ;
$$

i.e., $z_{n,(1)} \in(1,0)+\Gamma_{n,(1,-1)}$.

The next three lemmas state various estimates for the functions $a_{n, j}$ needed for the proof of Proposition 5.2.

Lemma 6.5. For $n \in[0, \infty)^{3}$ with at most one component less than 1 , we have

$$
a_{n, j}(x) \leq\left[1+c \operatorname{dist}\left(x, \Gamma_{n, j} \cap \Omega_{n}^{-}\right)\right]^{-1}, \quad x \in \Omega_{n}^{-}, \quad j \in J,
$$

with $c$ a positive constant which does not depend on $x, n$, or $j$.

Proof. We may assume that $x:=(u, v) \in R$, in particular that $u, v \in$ $[0,1 / 2]$. We consider each $j \in J$ separately and suppress all references to $n$.

(i) $j=(1,0)$. We have

$$
a_{j}(x)=\left|\frac{u}{1+u}\right|^{r}\left|\frac{u+v}{1+u+v}\right|^{t} \leq 1 / 2 .
$$

This proves (12) since $\operatorname{dist}\left(x, \Gamma_{n, j} \cap \Omega_{n}^{-}\right) \leq 1$. For the estimate, we have used the fact that $\min \{r, t\} \geq 1$. 


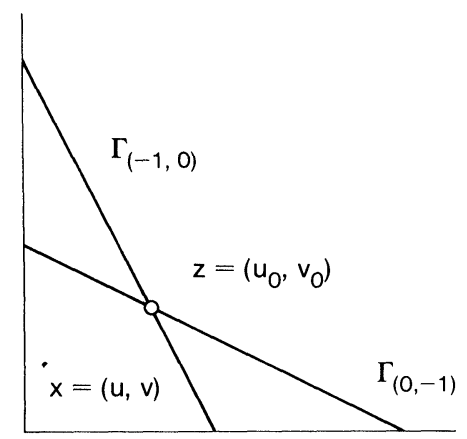

FIG. 6.5

The case $j=(0,1)$ is similar.

(ii) $j=(-1,0)$. Fig. 6.5 may be of help in following the argument. Let $i:=(0,-1)$ and let $z=:\left(u_{0}, v_{0}\right):=z_{(1)}$ be the intersection of the two curves $\Gamma_{j}$ and $\Gamma_{i}$. We consider two cases.

For $v \leq v_{0}$, there exists

$$
\varepsilon \geq \operatorname{dist}_{\infty}\left(x, \Gamma_{j} \cap \Omega^{-}\right)
$$

such that $(u+\varepsilon, v) \in \Gamma_{j}$, i.e., $a_{j}(u+\varepsilon, v)=1$. It follows that

$$
\begin{aligned}
a_{j}(u, v) & =a_{j}(u, v) / a_{j}(u+\varepsilon, v) \\
& =\left(\frac{u}{u+\varepsilon}\right)^{r}\left(\frac{1-u-\varepsilon}{1-u}\right)^{r}\left(\frac{u+v}{u+v+\varepsilon}\right)^{t}\left(\frac{1-u-v-\varepsilon}{1-u-v}\right)^{t} \\
& \leq\left(\frac{u+v}{u+v+\varepsilon}\right)^{\max \{r, t\}} \\
& \leq(1+\varepsilon)^{-1} .
\end{aligned}
$$

For $v \geq v_{0}$, we have $\operatorname{dist}_{\infty}\left(x, \Gamma_{j} \cap \Omega^{-}\right)=u_{0}-u$. After possibly increasing $v$ and therefore also increasing

$$
a_{j}(x)=\left(\frac{u}{1-u}\right)^{r}\left(\frac{u+v}{1-u-v}\right)^{t}
$$

we may assume that $x \in \Gamma_{i}$, i.e., $a_{i}(x)=1$. Using the fact that $a_{j}(z)=1=$ $a_{i}(z)$, we obtain

$$
a_{j}(x)=\frac{a_{j}(x) a_{i}(z)}{a_{j}(z) a_{i}(x)}=\left(\frac{u /(1-u)}{u_{0} /\left(1-u_{0}\right)}\right)^{r} /\left(\frac{v /(1-v)}{v_{0} /\left(1-v_{0}\right)}\right)^{s} .
$$


For $0 \leq p<q \leq 1 / 2$, we have

$$
\frac{q(1-p)}{p(1-q)}=1+\frac{q(1-p)-p(1-q)}{p(1-q)}=1+\frac{q-p}{p(1-q)} \geq 1+4(q-p) .
$$

This inequality implies that

$$
1 / a_{j}(x) \geq\left(1+4 r\left|u-u_{0}\right|\right)\left(1+4 s\left|\frac{v-v_{0}}{u-u_{0}}\right|\left|u-u_{0}\right|\right) .
$$

This proves the desired estimate for $a_{j}(x)$ in case $r \geq 1$. If $r<1$, we use the second factor of the product (13) together with the fact that $s \mid\left(v-v_{0}\right) /(u-$ $\left.u_{0}\right) \mid$ can be bounded below, uniformly in $n$ and $x$. Since both $(u, v)$ and $\left(u_{0}, v_{0}\right)$ lie on the curve

$$
\Gamma_{i}:\left(\frac{v}{1-v}\right)^{s}\left(\frac{u+v}{1-u-v}\right)^{t}=1,
$$

this last fact is established once we show that, on that curve,

$$
\min _{v_{0} \leq v \leq 1 / 2} s|d v / d u| \geq c
$$

for some positive $c$ independent of $n$. For this, a direct calculation yields

$$
1 /|s d v / d u|=\frac{1}{s}+\frac{1}{t} \frac{(u+v)(1-u-v)}{v(1-v)} \leq 1+\frac{1}{t v} \leq 1+\frac{1}{t v_{0}} .
$$

Since, for $(u, v) \in \Gamma_{i}$,

$$
s \ln \left|\frac{v}{1-v}\right|+t \ln \left|\frac{u+v}{1-u-v}\right|=0,
$$

and $u \in[0,1 / 2]$, we conclude that, for $v_{0} \leq 1 / 4$,

$$
t v_{0}=-\frac{s v_{0} \ln \left|v_{0} /\left(1-v_{0}\right)\right|}{\ln \left|\left(u_{0}+v_{0}\right) /\left(1-u_{0}-v_{0}\right)\right|} \geq v_{0} / \ln \left|\frac{1 / 2+v_{0}}{1 / 2-v_{0}}\right|,
$$

and this shows (14).

The case $j=(0,-1)$ is treated similarly.

(iii) $j=(1,-1)$. We have to show that

$$
\left|\frac{u+1}{u}\right|^{r}\left|\frac{1-v}{v}\right|^{s} \geq 1+c \operatorname{dist}\left((u, v), \Gamma_{j} \cap \Omega^{-}\right),
$$




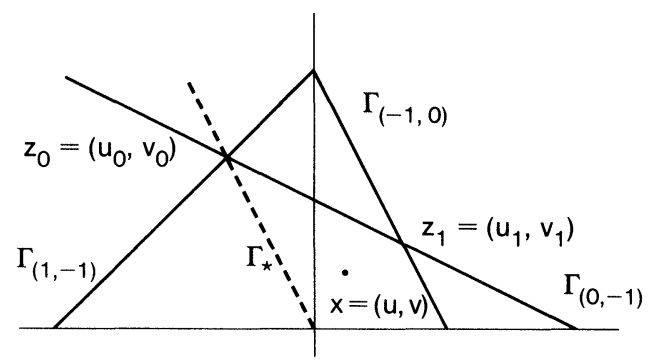

FIG. 6.6

and this is obvious for $r \geq 1$ in view of our assumption $(u, v) \in R \cap \Omega^{-}$. To prove (15) for $r<1$, let $i:=(-1,0)$ and consider the situation as depicted in Figure 6.6. We may assume that $x=(u, v) \in \partial \Omega \cap R$, since increasing $u$ decreases $|(u+1) / u|$ and increases $\operatorname{dist}\left(x, \Gamma_{j} \cap \Omega^{-}\right)$. For $x \in\left(\Gamma_{(-1,0)} \cup\right.$ $\left.\Gamma_{(0,-1)}\right) \cap \Omega^{-}$, we have

$$
\operatorname{dist}\left(x, \Gamma_{j} \cap \Omega^{-}\right)=\operatorname{dist}\left(x, z_{0}\right)=u-u_{0} .
$$

This follows because $\Gamma_{j}$ has nonnegative slope as a function of $u$ and passes through the point $(-1 / 2,1 / 2)$, while $\left(\Gamma_{(-1,0)} \cup \Gamma_{(0,-1)}\right) \cap \Omega^{-}$is contained in the triangle spanned by $(1 / 2,0),(1 / 2,1 / 2),(0,1 / 2)$. Also note that (cf. Figure 6.6)

$$
u_{0}=-u_{1}, \quad v_{0}-1 / 2=1 / 2-v_{1} \text {. }
$$

This is a consequence of the radial symmetry of the curves $\Gamma_{i}$ with respect to the point $-i / 2$. In Figure 6.6, the curve $\Gamma_{*}$ which passes through $z_{0}$ is meant to be the curve $\Gamma_{(-1,0)}-j$. For the proof of (15), we consider the two cases.

(a) $x \in \Gamma_{(-1,0)} \cap \Omega^{-}$; i.e., $u_{1} \leq u$. In this case, from $a_{(-1,0)}(x)=1$ we obtain the estimate

$$
\begin{aligned}
1 / a_{j}(x)-a_{(-1,0)}(x) / a_{j}(x) & =\left|\frac{u+1}{1-u}\right|^{r}\left|\frac{1-v}{v}\right|^{s}\left|\frac{u+v}{1-u-v}\right|^{t} \\
& \geq\left|\frac{1-v}{v}\right|\left|\frac{u+v}{1-u-v}\right| \\
& \geq 1+u,
\end{aligned}
$$

where we have used the fact that $s, t \geq 1$, and the last inequality is easily 
checked. Since

$$
\left|u-u_{0}\right| \leq|u|+\left|u_{0}\right| \leq 2|u|,
$$

this proves (15) for this case.

(b) $x \in \Gamma_{(0,-1)} \cap \Omega^{-}$, i.e., $0 \leq u \leq u_{1}=\left|u_{0}\right|$. First we assume that

$$
v_{0}-1 / 2 \geq\left|u_{0}\right| / 3 \text {. }
$$

Since $z_{0} \in \Gamma_{j}$, we have

$$
\begin{aligned}
a_{j}(x) & =a_{j}\left(z_{0}\right) / a_{j}(x) \\
& =\left|\frac{(u+1) / u}{\left(u_{0}+1\right) / u_{0}}\right|^{r}\left|\frac{(1-v) / v}{\left(1-v_{0}\right) / v_{0}}\right|^{s} \\
& \geq\left|1+\frac{\left(v_{0}-v\right) /\left(v v_{0}\right)}{\left(1-v_{0}\right) / v_{0}}\right| \\
& \geq 1+\left(v_{0}-v\right) .
\end{aligned}
$$

In view of $\left|u-u_{0}\right| \leq 2\left|u_{0}\right| \leq 6\left(v_{0}-1 / 2\right) \leq 6\left(v_{0}-v\right)$, this proves (15) under the assumption (18).

Next, suppose that

$$
v_{0}-1 / 2 \leq\left|u_{0}\right| / 3 \text {. }
$$

We claim that

$$
\left|\left(u_{0}+1\right) / u_{0}\right|^{r} \geq 1+c\left|u_{0}\right|
$$

and this finishes the proof of (15) for this case, in view of (16) and the inequalities

$$
1 / a_{j}(x) \geq\left|\frac{u+1}{u}\right|^{r} \geq\left|\frac{u_{0}+1}{u_{0}}\right|^{r} \geq 1+c\left|u_{0}\right| \geq 1+(c / 2)\left|u-u_{0}\right| .
$$

To prove (20), we use the fact that $z_{0} \in \Gamma_{*}=\Gamma_{(-1,0)}-j$ and therefore

$$
\left|\left(u_{0}+1\right) / u_{0}\right|^{r}\left|\left(u_{0}+v_{0}\right) /\left(1-u_{0}-v_{0}\right)\right|^{t}=1
$$

Solving for $r$, we obtain

$$
\begin{aligned}
r & =t \frac{\ln \left|\left(u_{0}+v_{0}\right) /\left(1-u_{0}-v_{0}\right)\right|}{\ln \left|\left(u_{0}+1\right) / u_{0}\right|} \\
& \geq-\ln \left|\frac{1 / 2-(2 / 3)\left|u_{0}\right|}{1 / 2+(2 / 3)\left|u_{0}\right|}\right| / \ln \left|\left(u_{0}+1\right) / u_{0}\right|
\end{aligned}
$$


Here we used the assumption (19) and the fact that $t \geq 1$. Therefore, we have

$$
r \ln \left|\left(u_{0}+1\right) / u_{0}\right| \geq \ln \left|\frac{1+(4 / 3)\left|u_{0}\right|}{1-(4 / 3)\left|u_{0}\right|}\right| \geq \ln |1+(4 / 3)| u_{0}||
$$

which establishes (20). This completes the case $j=(1,-1)$.

The case $j=(-1,1)$ is treated similarly.

From the statement of Proposition 5.2, recall the definition

$$
J^{\prime}:=\mathbf{A}^{*}(1,1)=\{ \pm(1,1), \pm(2,-1), \pm(-1,2)\} .
$$

Lemma 6.6. For $n \in[0, \infty)^{3} \cap N, x \in \Omega_{n}^{-}$, and $j \in J^{\prime}$

$$
a_{n, j}(x) \leq[1+C \operatorname{dist}(x,-j / 2)]^{-1},
$$

with $C$ a positive constant which does not depend on $n, x$, or $j$.

Proof. Assume without loss that $x=(u, v) \in R$.

First we consider $j=(-1,-1)$. For example, assume that

$$
1 / 2-u=\operatorname{dist}_{\infty}(x,(1,1) / 2)=: \varepsilon .
$$

Then we have

$$
\begin{aligned}
a_{(-1,-1)}(x) & =\left|\frac{u}{1-u}\right|^{r}\left|\frac{v}{1-v}\right|^{s}\left|\frac{u+v}{2-u-v}\right|^{t} \\
& \leq\left|\frac{1 / 2-\varepsilon}{1 / 2+\varepsilon}\right|^{r}\left|\frac{1-\varepsilon}{1+\varepsilon}\right|^{t} \\
& \leq 1 /(1+\varepsilon) .
\end{aligned}
$$

In the remaining cases $j \in J^{\prime} \backslash(-1,-1)$, we have $\operatorname{dist}_{\infty}(-j / 2, R) \leq 2$ and therefore it is sufficient to bound $a_{j}(x)$ by an absolute constant less than 1 . This is straightforward, using the fact that $a_{(-1,0)}(x), a_{(0,-1)}(x) \leq 1$. We list only the estimates:

For $j=(1,1)$,

$$
\left|\frac{u}{u+1}\right|^{r}\left|\frac{v}{v+1}\right|^{s}\left|\frac{u+v}{u+v+2}\right|^{t} \leq 3^{-r} 3^{-s} 2^{-t} .
$$

For $j=(2,-1)$,

$$
\begin{aligned}
a_{(2,-1)}(x) & \leq a_{(2,-1)}(x) / a_{(0,-1)}(x) \\
& =\left|\frac{u}{2+u}\right|^{r}\left|\frac{1-u-v}{2-u-v}\right|^{t} \\
& \leq 5^{-r} 2^{-t} .
\end{aligned}
$$


For $j=(-2,1)$,

$$
\begin{aligned}
a_{(-1,2)}(x) & \leq a_{(-1,2)}(x) / a_{(-1,0)}(x) \\
& =\left|\frac{v}{2+v}\right|^{s}\left|\frac{1-u-v}{2-u-v}\right|^{t} \\
& \leq 5^{-s} 2^{-t} .
\end{aligned}
$$

The remaining two cases, $j=(-2,1),(1,-2)$ are similar.

Lemma 6.7. For $n \in N, x \in \Omega_{n}^{-}$, and $j \in \mathbf{Z}^{2} \backslash\left(0 \cup J \cup J^{\prime}\right)$,

$$
a_{n, j}(x) \leq[1+C|j|]^{-1},
$$

with $C$ a positive constant which does not depend on $n, x$, or $j$.

Proof. Let $j=(k, l)$ and $x=(u, v)$ and, without loss of generality, let $x \in R$; hence $u, v \in[0,1 / 2]$. We consider several cases.

(i) $k, l \neq 0,-1$, and $k+l \neq 0,-1,-2$. Then

$$
\begin{gathered}
\left|\frac{u}{u+k}\right| \leq(1+|k|)^{-1}, \quad\left|\frac{v}{v+l}\right| \leq(1+|l|)^{-1}, \\
\left|\frac{u+v}{u+v+k+l}\right| \leq(1+|k+l| / 3)^{-1} .
\end{gathered}
$$

Using the facts that

$$
(1+p)^{-1}(1+q)^{-1} \leq(1+p+q)^{-1} \text { for } p, q \geq 0
$$

and

$$
|p|+|p+q| \geq \max \{|p|,|q|\},
$$

we see that the product of any two of the above lefthand terms is bounded by

$$
\left(1+|j|_{\infty} / 3\right)^{-1} \text {. }
$$

Since at most one of $r, s, t$ is less than 1 , this yields

$$
a_{n, j}(x) \leq(1+|k|)^{-r}(1+|l|)^{-s}(1+|k+l| / 3)^{-t} \leq\left(1+|j|_{\infty} / 3\right)^{-1} .
$$

(ii) $j=(0,-2)$. Since $x \in \Omega_{n}$, we have

$$
a_{n,(0,-1)}(x)=\left|\frac{v}{1-v}\right|^{s}\left|\frac{u+v}{1-u-v}\right|^{t}<1
$$


and therefore

$$
\begin{aligned}
a_{n, j}(x) & <a_{n, j}(x) / a_{n,(0,-1)}(x) \\
& =\left|\frac{1-v}{2-v}\right|^{s}\left|\frac{1-u-v}{2-u-v}\right|^{t} \\
& \leq 2^{-s} 2^{-t} \\
& \leq 1 / 2 \\
& =\left(1+|j|_{\infty} / 2\right)^{-1} .
\end{aligned}
$$

(iii) $k=0, l \neq-2,-1,0$. Here

$$
\begin{aligned}
a_{n, j}(x) & =\left|\frac{v}{v+l}\right|^{s}\left|\frac{u+v}{u+v+l}\right|^{t} \\
& \leq(1+|l|)^{-s}(1+|l| / 3)^{-t} \\
& \leq\left(1+|j|_{\infty} / 3\right)^{-1} .
\end{aligned}
$$

(iv) $k=-1, l \neq-1,0,1,2$. Here

$$
\begin{aligned}
\left|\frac{u}{1-u}\right|^{r}\left|\frac{v}{v+l}\right|^{s}\left|\frac{u+v}{u+v+l-1}\right|^{t} & \leq 1 \cdot(1+|l|)^{-s}(1+|l-1| / 3)^{-t} \\
& \leq\left(1+|j|_{\infty} / 5\right)^{-1} .
\end{aligned}
$$

(v) $k=0,-1$ and $l=0,-1$. Treated in analogy to cases (ii)-(iv).

(vi) $l=-k, k \neq-1,0,1$. Here

$$
\begin{aligned}
a_{n, j}(x) & =\left|\frac{u}{u+k}\right|^{r}\left|\frac{v}{v-k}\right|^{s} \\
& \leq(1+|k|)^{-r}(1+|k|)^{-s} \\
& \leq\left(1+|j|_{\infty}\right)^{-1} .
\end{aligned}
$$

(vii) $l=-k-1, k \neq-2,-1,0,1$. Here

$$
\begin{aligned}
a_{n, j}(x) & <a_{n, j}(x) / a_{n,(-1,0)}(x) \\
& \leq\left|\frac{1-u}{u+k}\right|^{r}\left|\frac{v}{v-k-1}\right|^{s} \\
& \leq|k|^{-r}(1+|k-1|)^{-s} \\
& \leq(1+|k| / 2)^{-1} \\
& \leq\left(1+|j|_{\infty} / 3\right)^{-1} .
\end{aligned}
$$


(viii) $l=-k-2, k \neq-2,-1,0$. Here

$$
\begin{aligned}
a_{n, j}(x) & \leq a_{n, j}(x) / a_{n,(-1,0)}(x) \\
& =\left|\frac{u-1}{u+k}\right|^{r}\left|\frac{v}{v-k-2}\right|^{s}\left|\frac{u+v-1}{u+v-2}\right|^{t} \\
& \leq(1+|k| / 3)^{-1} \\
& \leq\left(1+|j|_{\infty} / 9\right)^{-1} .
\end{aligned}
$$

This covers all $j \in \mathbf{Z}^{2} \backslash\left(0 \cup J \cup J^{\prime}\right)$.

Proof of Proposition 5.2. Lemmas 5-7 prove (5.4) for $n \in N \cap[0, \infty)^{3}$, with the constants independent of $n$. Since $\Omega_{n}$ depends continuously on $n \in N$ (by Proposition 5.1), this proves (5.4) for all $n \in N$.

Proof of Proposition 5.4. To prove (5.6), we consider three cases.

(i) $j \in J$. From the geometry of the set $\Omega_{n}=\Omega_{n^{\prime}}$ (cf. Figure 5.1 which gives a qualitatively accurate description of the general situation) and, in particular, from the estimates of the slopes of the curves $\Gamma_{n, j}$, we can see that in this case

$$
\operatorname{dist}\left(x, \partial \Omega_{n}\right)=\operatorname{dist}\left(x^{\prime}, \Gamma_{n, j} \cap \Omega_{n}^{-}\right) .
$$

Therefore, (5.6) is a consequence of Lemma 6.5 in this case.

(ii) $j \in J^{\prime}$. For example, assume that $x^{\prime} \in R \cap \Omega_{n}$. Then, for $j \in J^{\prime} \backslash$ $(-1,-1)$, we have

$$
\operatorname{dist}_{\infty}\left(x^{\prime},-j / 2\right) \geq 1 / 2
$$

and (5.6) follows from Lemma 6.6 since dist $t_{\infty}\left(x, \partial \Omega_{n}\right) \leq C$.

It remains to consider the case $j=(-1,-1)$. From the bounds on the slopes of the curves $\Gamma_{n,(1,0)}, \Gamma_{n,(0,1)}$, we see that $\Omega_{n}$ lies in the half space

$$
\left\{y: y^{*}(1,1) \geq-\left(u_{0}, v_{0}\right)^{*}(1,1)\right\}
$$

where $\left(u_{0}, v_{0}\right):=z_{(1)}$ is the point of intersection of the curves $\Gamma_{n,(-1,0)}$ and $\Gamma_{n,(0,-1)}$. Since $\Omega_{n} \cap R \subseteq[0,1 / 2]^{2}$, it follows that, for $x^{\prime} \in \Omega_{n} \cap R$,

$$
\operatorname{dist}_{1}\left(x, \partial \Omega_{n}\right)=\operatorname{dist}_{1}\left(x^{\prime},-z_{(1)}+(1,1)\right) .
$$

Here, dist $t_{1}$ denotes the $l_{1}$-distance. Moreover, in view of

$$
\left[-z_{(1)}+(1,1)\right]-(1 / 2,1 / 2)=(1 / 2,1 / 2)-z_{(1)},
$$


we have

$$
\begin{aligned}
\operatorname{dist}_{1}\left(x^{\prime},-z_{(1)}+(1,1)\right) & =\operatorname{dist}_{1}\left(x^{\prime}, z_{(1)}\right)+2 \operatorname{dist}_{1}\left(z_{(1)},(1 / 2,1 / 2)\right) \\
& \leq 2 \operatorname{dist}_{1}\left(x^{\prime},-j / 2\right) .
\end{aligned}
$$

This, together with (21) and Lemma 5, proves (5.6) for this case.

(iii) $j \in \mathbf{Z}^{2} \backslash\left(0 \cup J \cup J^{\prime}\right)$. In this case, (5.6) follows from Lemma 7 since, for any $j \neq 0$,

$$
\operatorname{dist}\left(x, \partial \Omega_{n}\right) \leq C|j|
$$

This completes the proof of Proposition 5.4.

\section{REFERENCES}

[BH $\left.{ }_{1}\right]$. C. DE BooR and K. Hollig, B-splines from parallelepipeds, J. d'Analyse Math., vol. 42 (1983), pp. 99-115.

$\left[\mathrm{BH}_{2}\right] . \ldots$ B Bivariate box splines and smooth pp functions on a three-direction mesh, J. Comput. Appl. Math. vol. 9 (1983), pp. 13-28.

[MRR]. M.J. MARSDEN, F.B. RichaRDS and S.D. RiemensChNeIder, Cardinal spline interpolation operators on $l_{p}$ data, Indiana Math. J., vol. 24 (1975), pp. 677-689.

[R]. S.D. RIEMENSCHNEIDER, Convergence of interpolating cardinal splines: Power growth, Israel J. Math., vol. 23 (1976), pp. 339-346.

[S $\mathrm{S}_{1}$ ]. I.J. SchoenBerg, Contribution to data smoothing, Quart. Appl. Math., vol. 4 (1946), pp. 45-99 and 112-141.

$\left[\mathrm{S}_{2}\right]$. Notes on spline functions III, On the convergence of the interpolating cardinal splines as their degree tends to infinity, Israel J. Math., vol. 16 (1973), pp. 87-93.

$\left[\mathrm{S}_{3}\right]$ _ _ Cardinal spline interpolation, SIAM, Philadelphia, 1973.

\section{UNIVERSITY OF WISCONSIN}

MADISON, WISCONSIN

\section{UNIVERSITY OF ALBERTA}

EDMONTON, CANADA 